\title{
CARACTERIZACIÓN LABORAL DEL INSTRUMENTADOR QUIRÚRGICO
}

\author{
Luz Dary Castro*
}

\section{Resumen}

Introducción: cuando la instrumentación quirúrgica se volvió carrera profesional en 2000, los campos de acción laboral se diversificaron hacia las áreas administrativa, gerencial, docente y gestión comercial. Objetivo: caracterizar el desempeño laboral de los profesionales egresados de la Fundación Universitaria de Ciencias de la Salud titulados entre 2006 y 2010. Materiales y métodos: estudio descriptivo de corte transversal. Se aplicó una encuesta a 231 egresados de instrumentación quirúrgica para conocer las características laborales en desempeño, salario y tiempo que le tomó conseguir empleo. Resultados: el área con mayor ejercicio profesional es el asistencial $55.6 \%$, seguida de asesoría y gestión comercial $20.4 \%$, administrativa $8.2 \%$, el $0.5 \%$ laboran en la docencia y el $15.3 \%$ están en áreas combinadas: asistencial y docente, asistencial y gestión comercial, administrativa y asesoría de gestión, gestión comercial y docencia. Conclusiones: el resultado sugiere crear espacios de discusión para reformar la malla curricular y así formarlos en áreas administrativas, gerenciales, de mercadeo y salud pública. La inserción laboral de los egresados en el campo laboral se considera rápida ( $77 \%$ en menos a seis meses).

Palabras clave: perfil laboral, empleo, remuneración.

\section{J OB CHARACTERIZATION OF SURGICAL INSTRUMENT TECHNICIANS}

\section{Abstract}

Introduction: when surgical instrument technician degree became a professional career in 2000, work activities diversified to areas such as, administration, management, teaching and commercial management.

Objective: to characterize jobs performed by graduates of Fundación Universitaria de Ciencias de la Salud between 2006 and 2010. Materials and Methods: cross-sectional descriptive trial. A survey was applied to 231 surgical instrument technician graduates to determine the characteristics of the jobs they perform, salary and time to get a job. Results: most of them practiced in the health care area $55.6 \%$, followed by working as consultants and commercial managers $20.4 \%$, administrators $8.2 \%, 0.5 \%$ as teachers and $15.3 \%$ in combined areas such as: health care and teaching; health care and commercial managing; administration and management consultants; commercial managers and teaching. Conclusions: results suggest creating spaces to discuss a curriculum modification in order to prepare them in the administrative, managing, marketing and public heath areas. Insertion of these graduates into the job market is considered quick ( $77 \%$ in less than six months).

Key words: work profile, job, remuneration

Fecha recibido: julio 22 de 2013 - Fecha aceptado: junio 26 de 2014

* Instrumentadora quirúrgica, especialista en Gerencia Educativa. Instructor Asociado, Facultad de Instrumentación Quirúrgica, Fundación Universitaria de Ciencias de la Salud, Bogotá, DC, Colombia. 


\section{Introducción}

El programa de instrumentación quirúrgica se inició en Colombia en los años cincuenta con un nivel técnico; después el modelo cambió a formación tecnológica y en 1996 se estrenó el plan de estudios profesional en la Fundación Universitaria de Ciencias de la Salud, siendo esta la primera institución educativa en ofrecerlo.

Esta ocupación se ha desarrollado en esencia en el campo asistencial, orientada a la disminución de las infecciones intrahospitalarias, administración de centrales de esterilización y quirófanos, e implementación de estrategias comerciales y de mercadeo en la industria hospitalaria, logrando así posición laboral a nivel nacional e internacional a partir de la aplicación y vigilancia de los procesos de asepsia ${ }^{1}$, antisepsia y bioseguridad, contribuyendo al desarrollo rápido y eficiente del procedimiento quirúrgico en beneficio del paciente

La evolución del programa se ha dado gracias a los progresos en los campos de acción, identificados por la Asociación Colombiana de Facultades de Instrumentación Quirúrgica (ACFIQ), a partir de la formación integral del instrumentador quirúrgico, desde los saberes profesional, humanístico e investigativo, en los cuales se puede desempeñar en el área asistencial, administrativa y de salud pública, investigación, asesoría y gestión comercial, favoreciendo la diversificación laboral. ${ }^{1}$

Por su parte, el gobierno colombiano ha diseñado mecanismos de control encaminados a evaluar y mejorar la calidad de la educación superior en el país, creando políticas, estrategias y procesos consignados en el registro calificado y acreditación de alta calidad, donde manifiesta los lineamientos para las instituciones de educación superior (IES). ${ }^{2}$ En el factor 7 de los lineamientos para acreditación, característica número 38, está consignado el seguimiento al egresado así: "El programa hace seguimiento de la ubicación y de las actividades que desarrollan los egresados y se preocupa por verificar si estas actividades corresponden con los fines de la institución y del programa". En el mismo documento aparece la característica número 39: impac- to de los egresados en el medio social y académico. ${ }^{2}$ Para la Facultad de Instrumentación Quirúrgica de la Fundación Universitaria de Ciencias de la Salud representa un aspecto relevante conocer el posicionamiento laboral de sus egresados a través del índice de ocupación y los campos de acción en los cuales se desarrollan profesionalmente, con el fin de analizar la pertinencia curricular y la calidad del programa.

\section{Materiales y métodos}

Se realizó un estudio descriptivo de corte transversal tomando como referencia los lineamientos establecidos por el "Manual de Instrumentos y Recomendaciones sobre el Seguimiento de Egresados GRADUA2". ${ }^{3} \mathrm{Se}$ aplicó una encuesta a los egresados de la Facultad de Instrumentación Quirúrgica graduados entre los años 2006 y 2010, que indagaba sobre la actividad laboral, áreas de desempeño, remuneración salarial, tiempo transcurrido desde la obtención del título hasta la primera inserción laboral y tipo de contratación con la entidad. En total fueron 231 profesionales, algunos contactados por teléfono y otros mediante las redes sociales. Los datos suministrados fueron a una base de datos en Excel y analizada en Stata 10. Las variables cualitativas sexo, lugar de trabajo, área de desempeño laboral, formación complementaria, situación laboral actual, acceso al primer empleo, ingreso económico y tipo de vinculación laboral con la institución empleadora, se analizaron con frecuencias absolutas y relativas; la variable cuantitativa, edad, se resumió con medidas de tendencia central y de dispersión. Este trabajo fue aprobado por el comité de ética en investigación con seres humanos del Hospital de San José y FUCS.

\section{Resultados}

Se analizaron $\mathrm{N}=231$ sujetos con edad promedio de $26.1 \pm 3.5$ años; la situación laboral y caracterización de las áreas de desempeño laboral se encuentran en la Tabla 1. La remuneración económica se describe en la Tabla 2. Quienes trabajan en el campo asistencial $55.6 \%(\mathrm{n}=109)$ ganan de uno a dos millones de pesos colombianos, en asesoría y gestión comercial $20.4 \%$ $(n=40)$ de dos a cuatro millones, los del área adminis- 


\begin{tabular}{|c|c|c|}
\hline Características & n & $(\%)$ \\
\hline \multicolumn{3}{|l|}{ Situación laboral } \\
\hline empleado & 196 & $(84.8)$ \\
\hline desempleado & 35 & $(15.2)$ \\
\hline \multicolumn{3}{|l|}{ Áreas de desempeño laboral } \\
\hline asistencial & 109 & $(55.6)$ \\
\hline asesoría y gestión comercial & 40 & $(20.4)$ \\
\hline docencia & 1 & $(0.5 I)$ \\
\hline soporte técnico & 5 & $(2.5)$ \\
\hline administrativa & 16 & $(8.2)$ \\
\hline \multicolumn{3}{|l|}{ Áreas de desempeño laboral combinadas } \\
\hline asistencial y administrativa & 9 & $(4.6)$ \\
\hline asistencial y docencia & 7 & (3.6) \\
\hline asistencial y gestión comercial & 6 & $(3.1)$ \\
\hline administrativa y asesoría de gestión & 1 & $(0.5)$ \\
\hline gestión comercial y docencia & 1 & $(0.5)$ \\
\hline asistencial, docencia y gestión comercial & I & $(0.5)$ \\
\hline
\end{tabular}

\begin{tabular}{|l|c|c|}
\hline \multicolumn{3}{|c|}{ Tabla 2. Remuneración económica de los } \\
profesionales (pesos colombianos)
\end{tabular}

trativa $8.2 \%(\mathrm{n}=16)$ están entre dos y tres millones, mientras que $13.2 \%(\mathrm{n}=26)$ laboran en áreas combinadas con la asistencial, cuyo rango salarial está entre uno y dos millones de pesos y en soporte técnico 2.5 $\%(n=5)$ menor a un millón.

La caracterización laboral del instrumentador quirúrgico en cuanto a la contratación laboral con las instituciones empleadoras se encuentra descrita en la Tabla 3. Referente al tiempo trascurrido desde el mo- mento de la obtención del título a la primera inserción laboral, se encontró que el $77 \%(n=151)$ lo hizo en un tiempo menor a seis meses, $16 \%(\mathrm{n}=31)$ menor a un año, y $7 \%(\mathrm{n}=14)$ entre uno y dos años. La Tabla 4 presenta la educación complementaria realizada por el egresado. El 29.3\% ( $\mathrm{n}=23)$ de los profesionales continuó con su formación académica cursando diplomados y el $48.5 \%(n=114)$ no lo hizo. De acuerdo con la Figura 1, los egresados que continuaron con su formación académica y realizaron estudios de posgrado laboran en el área comercial, obtuvieron empleo por contrato directo en un tiempo menor de seis meses después de su grado, con salarios entre dos y cinco millones de pesos colombianos y cuentan con reconocimiento por su labor profesional.

Por su parte, los profesionales que se desempeñan en campos asistenciales y administrativos siguieron su formación complementaria en diplomados y educación continuada (asistencia a congresos, simposios y cursos cortos), tuvieron una inserción laboral más demorada (de 6 meses a 2 años), con contratos indirectos, devengando de uno a dos millones de pesos. Aseguraron que no tienen reconocimiento de su labor profesional.

Por otro lado, el estudio indagó las razones por las que 35 egresados están desempleados: el $42.8 \%(n=15)$ aduce que a pesar de iniciar el proceso de selección con las instituciones empleadoras no logró la inserción

\begin{tabular}{|c|c|c|c|c|c|}
\hline \multicolumn{6}{|c|}{ Clase de contratación } \\
\hline & & $\begin{array}{c}\text { Definido } \\
\text { n (\%) }\end{array}$ & $\begin{array}{c}\text { Indefinido } \\
\text { n (\%) }\end{array}$ & $\begin{array}{c}\text { Prestación } \\
\text { de servicios } \\
\text { n (\%) }\end{array}$ & $\begin{array}{l}\text { Total } \\
=196\end{array}$ \\
\hline \multirow{3}{*}{$\begin{array}{l}\text { Tipo de } \\
\text { contrato }\end{array}$} & directo & $25(12.7)^{*}$ & $88(44.9)^{* *}$ & $17(8.7)$ & 130 \\
\hline & indirecto & $4(2.1)$ & $42(2 I)$ & $14(7.1)$ & 60 \\
\hline & sociedad & $2(1)$ & $4(2.1)$ & $0(0)$ & 6 \\
\hline
\end{tabular}

*El 12.7\% labora con un contrato directo a término definido, es decir con renovación de contrato cada 3,6 ○ 12 meses. **En la caracterización laboral del instrumentador quirúrgico se resalta que el $44.9 \%(n=88)$ trabaja con la organización por medio de un contrato directo y contratación a término indefinido. 


\begin{tabular}{|l|c|c|}
\hline \multicolumn{3}{|c|}{ Tabla 4. Educación complementaria } \\
\hline Educación complementaria & $n$ & $(\%)$ \\
\hline diplomados & 70 & $(29.8)$ \\
\hline especialización & 21 & $(8.9)$ \\
\hline educación continuada* & 18 & $(7.6)$ \\
\hline especialización y doctorado & 1 & $(0.42)$ \\
\hline educación continuado y doctorado** & I & $(0.42)$ \\
\hline educación continuada y diplomado & 4 & $(1.7)$ \\
\hline diplomado y especialización & 5 & $(2.1)$ \\
\hline maestría & 1 & $(0.42)$ \\
\hline no ha realizado estudios de posgrado & 114 & $(48.5)$ \\
\hline
\end{tabular}

*Educación continuada se entiende como la asistencia a congresos y cursos cortos.** Doctorado en curso. laboral, $11.4 \%(\mathrm{n}=4)$ creó empresa en el sector comercial, $8.6 \%(n=3)$ inició estudios de pregrado en otra área de conocimiento diferente a la salud, $5.7 \%(\mathrm{n}=2)$ siguió especialización y no se insertó, $5.7 \%(\mathrm{n}=2)$ viajó a otro país y no ha ejercido la profesión y $25.7 \%$ (n=9) no contestó la pregunta.

De igual manera, se preguntó si desde la perspectiva de egresado consideraba que la formación académica de los instrumentadores quirúrgicos recibida en la FUCS debería cambiar en algo, ante lo cual el 62.8\% $(\mathrm{n}=22)$ manifestó que la formación académica no debe cambiar, mientras que $37.1 \%(\mathrm{n}=13)$ mencionó que se deben reforzar las áreas de gestión comercial, administración, salud ocupacional, docencia, investigación y soporte técnico. Así mismo, los profesionales contestaron que se deben perfeccionar los convenios con instituciones empleadoras para mejorar las oportunidades de inserción laboral de los egresados una vez terminado el pregrado y que se debe avanzar en la formación de un segundo idioma, específicamente inglés.

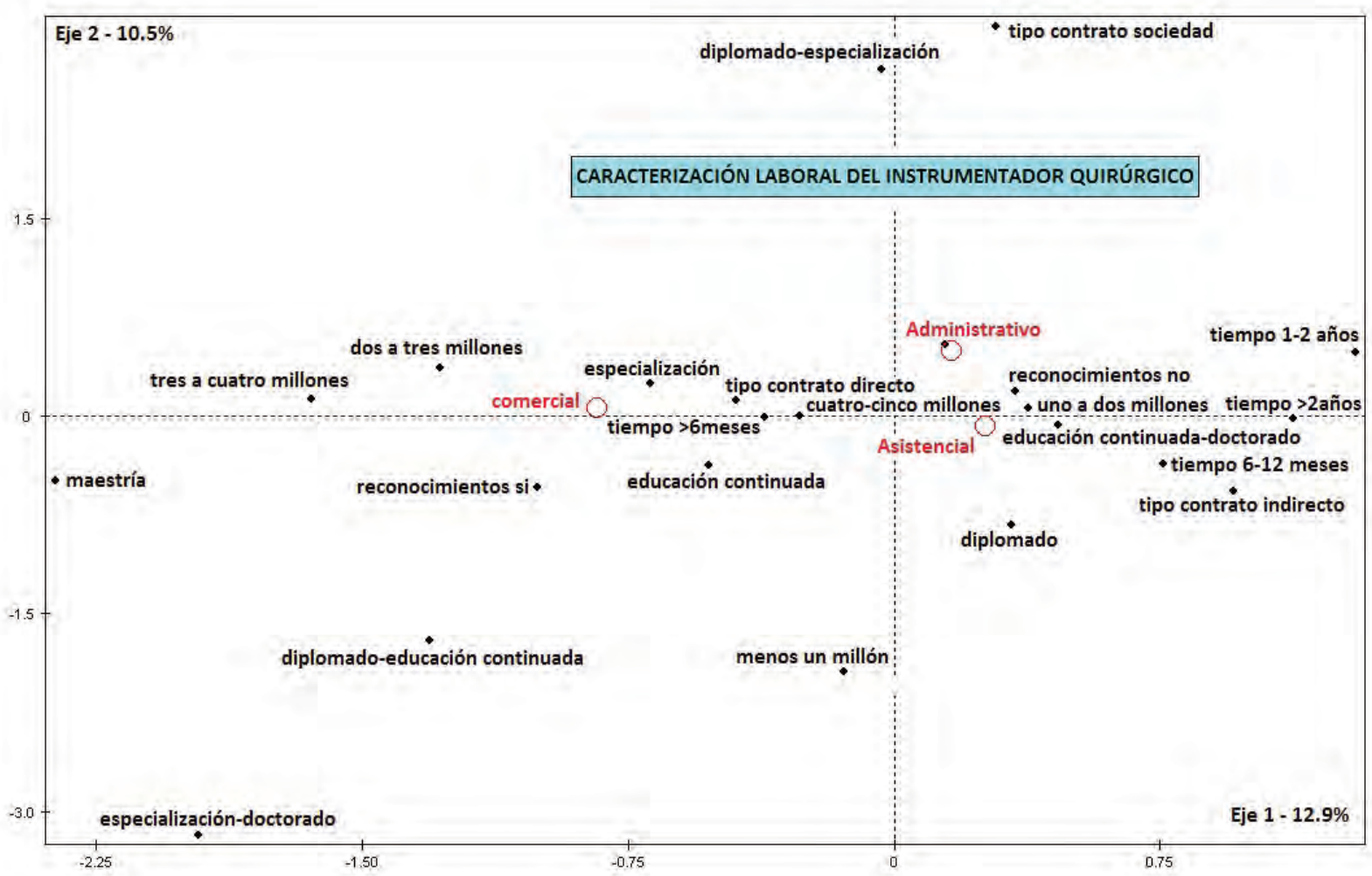

Figura I. Gráfica de correspondencias múltiples: muestra la relación entre las variables de caracterización laboral, las áreas de desempeño (color rojo) se agrupan con las variables de caracterización laboral (color negro), la cercanía entre las dos variables denota la caracterización laboral por área de desempeño. 


\section{Disc usión}

Según datos del Observatorio Laboral para la Educación, el $80 \%$ de los graduados a nivel educativo superior logra vincularse de manera formal al campo laboral, por lo cual obtienen mejores condiciones de empleo y garantías de seguridad social. ${ }^{4}$ El estudio en mención refleja que los egresados de instrumentación quirúrgica de la FUCS están alineados con el promedio nacional, con un $84.8 \%$ de ocupación laboral, evidenciando la pertinencia del programa profesional.

Si bien la profesionalización de la carrera permitió una apertura de los campos de acción laboral dado que los egresados accedieron a posgrados, el profesional no solo se desempeñó en el quirófano, sino en otras áreas de la salud. El estudio demuestra que la fortaleza de la formación académica de los instrumentadores quirúrgicos de la FUCS es el área asistencial y se refleja en la caracterización laboral del egresado, dado que el $55.6 \%$ de ellos trabaja en este área.

Teniendo en cuenta que las características del currículo descritas en el Proyecto Educativo Institucional (PEI) de la FUCS, son social y profesionalmente pertinentes, integradas e interdisciplinarias ${ }^{5}$, se hace innegable la importancia de crear espacios de discusión que permitan fortalecer reformas a la malla curricular, con el objetivo de formar a los profesionales en áreas administrativas, gerenciales, de gestión comercial y salud pública para favorecer la adquisición de competencias laborales que respondan a las necesidades en salud y de esta manera ampliar los campos de acción del profesional en instrumentación quirúrgica en un escenario laboral cambiante y competitivo.

En el documento del observatorio laboral para la Educación "Perfil académico y condiciones de empleabilidad de los graduados de educación superior 2002-2011" se contempla que el 90\% de los egresados de educación superior logran la inserción laboral en un tiempo menor a seis meses, comparado con los profesionales de instrumentación quirúrgica quienes se encuentran por debajo del promedio nacional, dado que el $77 \%$ se insertó en este mismo tiempo. En el mismo documento se resalta que el egresado de nivel universitario ganó en el 2010, en promedio \$1'525.3576, dato que coincide con la información encontrada en el estudio, teniendo en cuenta que el $72 \%$ de los profesionales en instrumentación quirúrgica están en el rango salarial de uno a dos millones de pesos. Por último, en el documento del Observatorio Laboral para la Educación "Resultados de las condiciones laborales de los graduados de la educación superior 2002-2011 (documento técnico)" se destaca el fortalecimiento de la formación de posgrado de los profesionales del país, que contribuye a la consolidación y mejora de las capacidades de investigación e innovación. ${ }^{7}$ Esta información contrasta con la realidad de los profesionales de instrumentación quirúrgica, puesto que de los sujetos encuestados $48.5 \%$ no cursó estudios de posgrado, mientras que los profesionales que continuaron con su formación académica y realizaron especializaciones se caracterizaron por tener mejores condiciones laborales, con una rápida inserción laboral, contratos directos, estables y remuneración más alta.

\section{Referencias}

1. Asociación Colombiana de Facultades de Instrumentación Quirúrgica. Exámenes de Calidad de la Educación Superior en Instrumentación Quirúrgica: guía de orientación [monografía en Internet]. Bogotá: ICFES; 2009 [citado 7 Jul 2014]. Disponible en: http://www.colombiaaprende.edu.co/html/mediateca/1607/articles-208990_recurso_1.pdf

2. Colombia. Ministerio de Educación Nacional. Sistema de aseguramiento de la Calidad de la Educación Superior. Bogotá: El Ministerio; 2006.

3. Programa ALFA de la comisión Europea. Proyecto GRADUA2 [monografía en Internet]. México: ITESM; 2006 [Citado 7 Jul 2014] Disponible en: http://www. almalaurea.it/sites/almalaurea.it/files/docs/universita/altro/red_gradua2.pdf

4. Colombia. Ministerio de Educación Nacional. Educación Superior una buena decisión. Bol Educ Super. 2012 Abr; 20: 10-4.

5. Fundación Universitaria de Ciencias de la Salud. Proyecto Educativo Institucional PEI. Bogotá: FUCS; 2008

6. Colombia. Ministerio de Educación Nacional. Perfil académico y condiciones de empleabilidad de los graduados de educación superior (2001 - 2012) y certificados de educación para el trabajo y el desarrollo humano (2010-2012): informe del Observatorio Laboral para la Educación. Bogotá: El Ministerio; 2006.

7. Colombia. Ministerio de Educación Nacional. Resultado de las condiciones laborales de los graduados de la educación superior. Bogotá: Observatorio Laboral para la Educación; 2012. 